\title{
Sophora flavescens Alkaloids and Corticosteroid Synergistically Augment IL- I0/IL-5 Ratio with Foxp3-Gene-Epigenetic Modification in Asthma PBMCs
}

Ying Song, ${ }^{1,2, *}$ Zhen-Zhen Wang, ${ }^{1,3 * *}$ Lixin Wang, (iD) ${ }^{4, *}$ Paul Faybusovich, ${ }^{2}$ Kamal Srivastava, ${ }^{3,5}$ Changda Liu, ${ }^{1,2}$ Jody Tversky, ${ }^{6}$ David Dunkin, ${ }^{7}$ Paula Busse, ${ }^{8}$ Xianqing Ren, (iD) ${ }^{9}$ Rachel Miller, ${ }^{8}$ Mingsan Miao,' Xiu-Min Li, $\mathrm{Li}^{3,10}$

'Academy of Chinese Medicine, Henan University of Chinese Medicine, Zhengzhou, People's Republic of China; ${ }^{2}$ Department of Pediatrics, Division of Allergy and Immunology, Icahn School of Medicine at Mount Sinai, New York, NY, USA; ${ }^{3}$ Department of Pathology, Microbiology and Immunology, New York Medical College, Valhalla, NY, USA; ${ }^{4}$ Integrated TCM \& Western Medicine Department, Shanghai Pulmonary Hospital Affiliated to Tongji University, Shanghai, People's Republic of China; ${ }^{5} \mathrm{General}$ Nutraceutical Technology LLC, Elmsford, NY, USA; ${ }^{6}$ The Department of Medicine, Division of Allergy and Clinical Immunology, Johns Hopkins Asthma and Allergy Center, Baltimore, Maryland, USA; ${ }^{7}$ Department of Pediatrics, Division of Gastroenterology and Nutrition, Icahn School of Medicine at Mount Sinai, New York, NY, USA; ${ }^{8}$ Division of Clinical Immunology, Department of Medicine, Icahn School of Medicine at Mount Sinai, New York, NY, USA; 'The First Affiliated Hospital of Henan University of TCM, Zhengzhou, People's Republic of China; ${ }^{10}$ Department of Otolaryngology, New York Medical College, Ardsley, NY, USA

*These authors contributed equally to this work
Background: It has been demonstrated that ASHMI (antiasthma-simplified herbal medicine intervention) can improve airway function and reduce inflammation in human asthmatic patients with high safety and tolerability. In addition, ASHMI significantly suppresses Th2 cytokine production and increases Th1 cytokine production in treating asthma.

Objective: Allergic asthma is associated with dysregulation of cytokines. We focused on IL-5 and IL-10 as signature Th2 and Treg cytokines to characterize ASHMI immunomodulatory components.

Methods: The effects of ASHMI and individual herbal constituents on IL-5 and IL-10 production by PBMCs from asthmatic subjects were determined ex vivo. Sophora flavescens (SF)-F2, containing alkaloid compounds, effects on PBMC IL-10 and IL-5 production in the presence or absence of dexamethasone (Dex), and on DNA methylation levels at the foxp 3 gene promoter were determined.

Results: The ratio of anti-CD3/CD28 stimulated IL-10/IL-5 production by PBMCs from asthmatic subjects was significantly reduced compared to healthy subjects. In PBMCs from asthmatic subjects, ASHMI significantly reduced IL-5 production and increased IL-10 secretion in a dose-dependent manner $(\mathrm{p}<0.05-0.01)$. SF-F2 was most effective in increasing IL-10, whereas SF-F4 (flavonoid compounds) was most effective in suppressing IL-5 production. Dex-treated PBMCs from asthma subjects showed a trend of increasing ratio of IL-10/IL-5 while demonstrating reduced levels in both IL-5 and IL-10 ( $p<0.05)$. Co-culture with Dex and SF-F2 significantly prevented Dex suppression of IL-10, while retained Dexsuppression of IL-5 production, and increased IL-10/IL-5 ratio by Dex. Co-culture with SFF2 and Dex significantly reduced DNA methylation levels at the foxp3 gene promoter at $\mathrm{CpG}^{-126}$.

Conclusion: The SF alkaloid-rich fraction may be responsible for ASHMI induction of IL10 production by PBMCs and plays a synergistic effect with Dex for augmenting IL-10/IL-5 ratio.

Keywords: Sophora flavescens, IL-10/IL-5 ratio, asthma, foxp3 methylation, synergistic effect

\section{Introduction}

It is well known that atopic asthma is associated with activation of a TH2 type of T cell in the airway. ${ }^{1,2}$ Expression of TH2 cytokines could be related to the activity of the disease, symptom scores, airway eosinophilia, and bronchial hyper-responsiveness.
Professor of Pathology, Microbiology and Immunology, New York Medical College, Valhalla, NY

Email XiuMin_Li@NYMC.edu 
They increase after allergen challenge and decrease with corticosteroid treatment of asthma. ${ }^{1,3}$ These reactions are normally suppressed by regulatory $\mathrm{T}$ cells, which maintain airway tolerance. The anti-inflammatory regulatory cytokine IL-10 plays a central role in the induction of tolerance. ${ }^{4}$ Studies have shown that Th2-skewed responses dominate in allergic asthma, characterized by IL-4, IL-13 and IL-5 production, ${ }^{5}$ whereas a $\mathrm{T}$ regulatory response, characterized by IL- 10 and TGF- $\beta$ production, inhibits the Th 2 response and induces immune tolerance to harmless environmental antigens. $^{6-9}$

For the treatment of allergic inflammation, the aim is to downregulate allergen-specific T helper 2 (Th2) responses and the associated clinical symptoms. Indeed, previous studies have shown that IL-10-secreting T cells in the airways were able to reduce Th2-type inflammation and airway hyperreactivity (AHR). ${ }^{9}$ Decreased IL-5 and/or increased IL-10 production by mononuclear cells in asthma patients who are in remission have been reported. ${ }^{10}$ Tomiita et al demonstrated the association of IL-10/IL-5 ratio with remission in asthmatic patients and further proposed that decreased IL- 5 production is due to the enhanced production of inhibitory or regulatory cytokines, such as IL-10. ${ }^{11-13}$ The PBMC in vitro allergen challenge model system has been widely used to evaluate the molecular effects of in vitro treatment. ${ }^{14}$ Therefore, in this study, we determined the effect of various regiments on the levels of IL-5 and IL-10 production and the ratio of IL-10/IL-5 in stimulated peripheral blood mononuclear cells (PBMCs) from subjects with asthma. Moreover, interferon- $\gamma$ (IFN- $\gamma$ ) secreted by Th1 cells exerts inhibitory effects on Th2 differentiation. ${ }^{12}$ The increase of IFN- $\gamma$ and reduce of IL- 4 and IL- 5 by CD4+ T-cells have been reported in peripheral T-cells following specific immunotherapy, suggesting a switching from $\mathrm{Th} 2$ to Th1 response. ${ }^{15}$ Therefore, the production of IFN- $\gamma$ by PBMCs from asthmatic patients was evaluated after treatment in this study.

Several current treatment options for allergy exist, including pharmacotherapy with immunosuppressive agents and allergen-specific immunotherapy. ${ }^{16}$ At present, inhaled corticosteroids (ICS) remain the cornerstone of pharmacologic therapy. ${ }^{17}$ However, moderate-to-high doses of corticosteroids with long-term use are associated with unfavorable side effects, including hyperglycemia, osteoporosis, hypertension, mood disturbances, weight gain and the complications associated with generalized immunosuppression. ${ }^{18,19}$ Moreover, corticosteroids have been reported to limit beneficial $\mathrm{T}$ cell responses such as eliminating the development of IL-10-producing Treg cells, which could enhance subsequent Th2 responses and aggravate the long-term course of asthma. ${ }^{20}$ Effective allergen immunotherapy can result in increased Treg cells and the suppression of Th2 cytokine production. Altering the cytokine environment with immunotherapy has been shown to increase Treg activity as well as IL10 and IFN- $\gamma$ production. ${ }^{21-23}$ Together, these changes suppress the allergenic Th2 response and increase the Th1 response, creating an immune tolerant environment. However, allergen immunotherapy is not effective for all asthma patients and requires a lengthy duration of treatment-typically $3-5$ years. ${ }^{24}$ In light of these barriers, continued research is needed to develop immune modulators to fill the gaps in asthma therapy. ${ }^{24}$

Anti-Asthma Simplified Herbal Medicine Intervention (ASHMI) is an extract of three Chinese herbal medications, Ganoderma lucidum(GL), Sophora flavescens (SF), and Glycyrrhiza uralensis (GU), that has been shown to have immunomodulatory effects in both animal models and humans. ${ }^{25-27}$ In murine models, ASHMI suppresses Th2 cytokine production and increases Th1 cytokine production. ${ }^{26}$ In a human study of moderate-to-severe asthma, we found that a combination of ASHMI and prednisone improved clinical symptoms, increased lung function, and inhibited Th2 cytokines, increased IFN- $\gamma$ levels, indicating that ASHMI might be a potential immune modulator. ${ }^{27}$ Previous studies have shown that the herbal constituents of ASHMI have been identified to specifically target the various mechanisms involved in the pathogenesis of asthma. ${ }^{28-33}$ For example, GL triterpenoid fractions and compounds inhibit TNF- $\alpha$ production by murine macrophages and human PBMCs from asthma patients. ${ }^{31} \mathrm{SF}$ and its flavonoid fractions inhibit acetylcholine-induced airway smooth muscle contraction. ${ }^{32}$ Interestingly, a recent study showed that 7,4'-dihydroxyflavone, the eotaxin/CCL11 inhibitor isolated from Glycyrrhiza uralensis (GU), prevented Dex-induced paradoxical adverse effects on eotaxin production. ${ }^{33}$ However, it remains unknown whether ASHMI enhances IL-10 production while inhibiting Th2 cytokine production and, if so, whether it prevents Dex-induced suppression of IL-10 production and the nature of the underlying mechanisms.

DNA methylation as a type of epigenetic modifications is generally associated with transcriptional repression. ${ }^{34}$ It is known that changes in the methylation pattern of the foxp 3 gene occur during $\mathrm{T}$ cell activation in asthma, ${ }^{35}$ and 
a study on asthmatic discordant monozygotic twins demonstrated that impaired Treg activity is associated with increased levels of $\mathrm{CpG}$ methylation within the foxp 3 gene locus. ${ }^{36}$ The foxp 3 gene expression is controlled by four CNSs (conserved non-coding sequences), of which one is in the promoter region, two are in the first intron, and the fourth is in the second intron. ${ }^{37}$ The promoter region is necessary for directly controlling gene expression, and the methylation in promoter are associated with the regulation of various gene expressions including AP1, FOXO, c-Rel, and NFAT. ${ }^{38}$ The CNS1-3 serves as an enhancer region or stabilizer compared with the promoter region. $^{38}$ Thus, we focused on investigating the $\mathrm{CpG}$ methylation sites in the promoter region.

In this study, we utilized human peripheral blood mononuclear cells (PBMC) from asthma subjects to determine ASHMI's immunomodulatory role on IL-5, IL-10, and IFN- $\gamma$ production and the ratio of IL-10/IL5. We further investigated the relationships between ASHMI and Tregs, DNA methylation, and asthmatic signature cytokines. We asked whether SF-F2 could prevent Dex suppression of IL-10 production or not and if so, if it co-cultures with SF-F2 and Dex, and whether DNA methylation levels are altered at the foxp3 gene promoter.

\section{Methods}

Informed written consent was obtained from subjects with physician-diagnosed current allergic asthma (ages 9-56, $\mathrm{n}=28$ ) and non-atopic healthy controls (age 12-44, $\mathrm{n}=12$ ). And informed written consent was obtained from the legal guardians of participants under 18 years of age. The patient characteristics are shown in Table 1. Per protocol, whole blood samples were collected, and PBMCs were isolated. This study has been approved by the Institutional Review Board of the Mount Sinai School of Medicine, which was conducted in accordance with the Declaration of Helsinki.

\section{Reagents}

Dexamethasone was purchased from Sigma-Aldrich (St. Louis, MO). Dynabeads ${ }^{\circledR}$ Human T-Activator CD3/ CD28 was purchased from Invitrogen (Grand Island, NY). Antibodies for ELISA were purchased from BD Pharmingen (San Diego, CA). DNA methylation reagents were purchased from Qiagen (Germantown, MD).
Table I Subject Characteristics

\begin{tabular}{|l|l|l|l|}
\hline \multicolumn{2}{|l|}{} & Asthma & $\begin{array}{l}\text { Non-Atopic Healthy } \\
\text { Controls }\end{array}$ \\
\hline No. individuals & 28 & 12 \\
\hline Male/Female & $15 / 13$ & $9 / 7$ \\
\hline Age (year) & $9-56$ & $12-44$ \\
\hline Mean Age (year) & 30 & 31 \\
\hline $\begin{array}{l}\text { Asthma } \\
\text { Severity }\end{array}$ & $\begin{array}{l}\text { Moderate } \\
\text { Severe } \\
\text { Unknown }\end{array}$ & $\begin{array}{l}23 \\
0\end{array}$ & $\begin{array}{l}\text { N/A } \\
\text { N/A } \\
\text { N/A }\end{array}$ \\
\hline $\begin{array}{l}\text { Asthma } \\
\text { Symptomatic }\end{array}$ & $\begin{array}{l}\text { Well- } \\
\text { controlled } \\
\text { Poorly } \\
\text { controlled }\end{array}$ & 25 & N/A \\
\hline Food Allergy & 3 & 19 & 0 \\
\hline Allergic Rhinitis & 24 & 0 \\
\hline
\end{tabular}

Abbreviation: NA, not applicable.

\section{Preparation of ASHMI and Its Fractions}

ASHMI was obtained from Sino-Lion Pharmaceutical Company (Shan Dong, China). The components of ASHMI were identified as the fruiting body of Ganoderma lucidum (GL), the roots of Sophora flavescens (SF), and the roots and rhizome of Glycyrrhiza uralensis (GU). Extensive quality control, analytical chemistry data and batch consistency pertaining to the formula have been published previously. ${ }^{25,27,39}$ Based on the ratio of individual raw herbs in ASHMI, the yield of individual herb extracts, and enhanced ease of product quality control, ASHMI was made by combining individual extracts of $\mathrm{GL}, \mathrm{SF}$ and GU at the following ratios- GL:SF:GU= 3.5: 4.5:2.0 in our laboratory. ${ }^{39}$ Fractions from ASHMI were extracted using a macroporous resin column (Amberlite* XAD-7 HP, Acros Organics, NJ) and graded concentrations of an ethanol and water elution were used to generate 4 fractions: 0\% ethanol F1 (polar), 20\% F2 (moderately polar), and a combination of $70 \% \mathrm{~F} 3$ (less polar or nonpolar), 95\% elution F4 (non-polar). SF showed stronger immunomodulatory activity on IL-10 and IL-5 production. Therefore, SF were further extracted and divided into SFF1-4. Fractions were further analyzed by highperformance liquid chromatography (HPLC) analysis (Figure 1). The concentrations of each SF fraction used were equivalent to the concentration of SF in ASHMI 

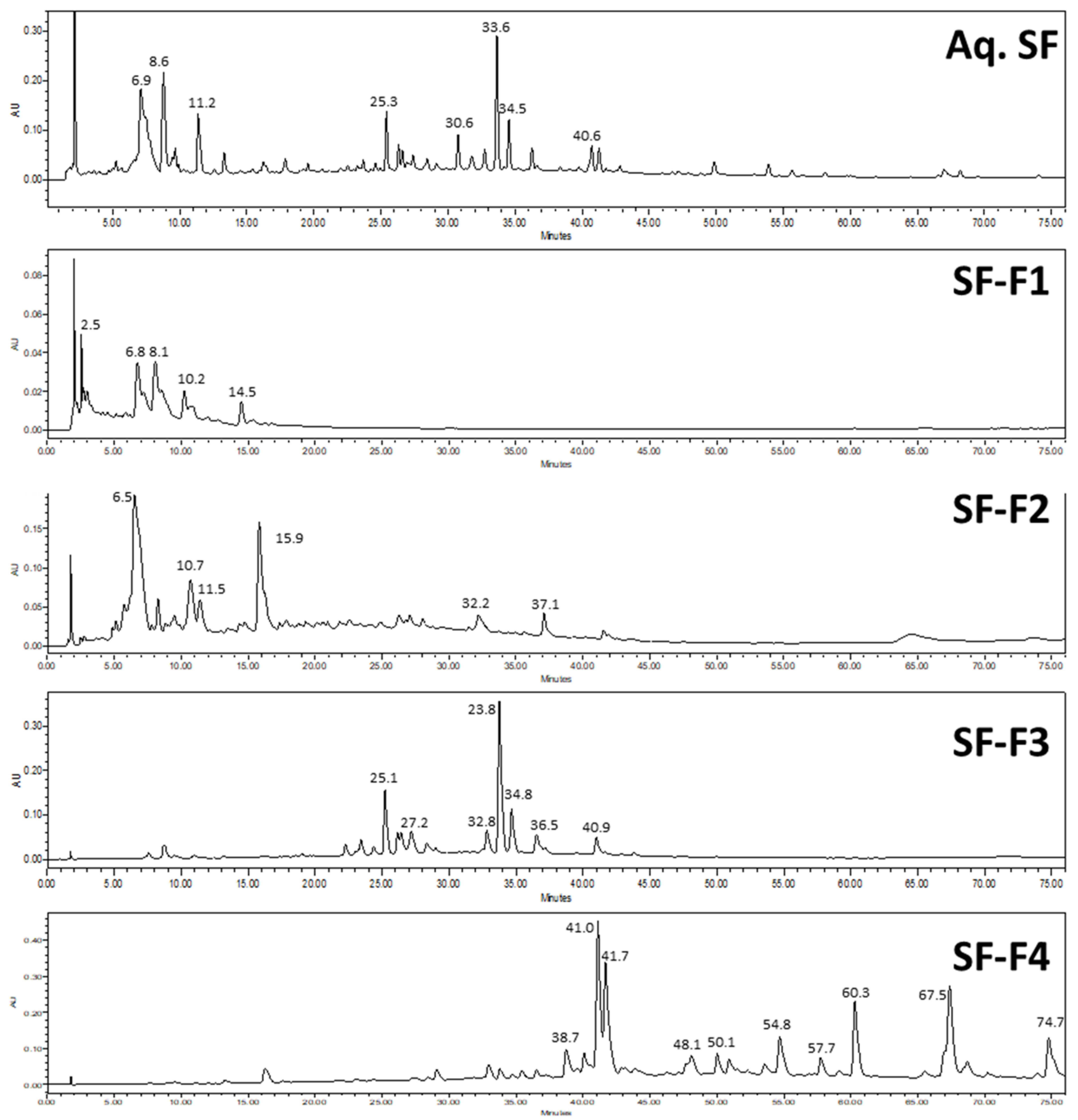

Figure I HPLC profiles of aqueous SF extract, SF-FI, SF-F2, SF-F3, SF-F4. SF-FI contained water-soluble compounds. SF-F2 contained alkaloids. SF-F3 and SF-F4 contained flavonoids and terpenoids.

$(56.3 \mu \mathrm{g} / \mathrm{mL}):$ SF-F1 $(17.3 \mu \mathrm{g} / \mathrm{mL}), \mathrm{SF}-\mathrm{F} 2(22.5 \mu \mathrm{g} / \mathrm{mL})$, SF-F3 $(9.2 \mu \mathrm{g} / \mathrm{mL})$, SF-F4 $(3.2 \mu \mathrm{g} / \mathrm{mL})$.

\section{PBMC Separation, Cell Culture and Cytokine Measurements}

PBMCs were separated from blood using a Ficoll gradient. Cells were then cultured at $4 \times 10^{5}$ cells per well in 96-well round bottom plates with either AIM-V media alone, AIM-
$\mathrm{V}$ with anti-CD3/28 Dynabeads ${ }^{\circledR}(5 \mu \mathrm{L} /$ well $/ 200 \mu \mathrm{L})$, or AIM-V with anti-CD3/28 Dynabeads ${ }^{\circledR}$ plus various doses of ASHMI or GL or SF or GU $(43.7 \mu \mathrm{g} / \mathrm{mL}, 56.3 \mu \mathrm{g} / \mathrm{mL}, 25$ $\mu \mathrm{g} / \mathrm{mL}$, respectively, equivalent to ASHMI at $125 \mu \mathrm{g} / \mathrm{mL}$ ) or fractions of SF. In a separate set of experiments, PBMCs were cultured in AIM-V with anti-CD3/28 alone, with antiCD3/28 plus Dex $\left(10^{-6} \mathrm{M}\right)$ or anti-CD3/28 plus SF fraction 2 (SF-F2, $22.5 \mu \mathrm{g} / \mathrm{mL}$ ) or with a combination of all three 
reagents. All cultures were incubated at $37^{\circ} \mathrm{C}$ for 3 days after which culture supernatants were analyzed by ELISA for the level of IL-5 and IL-10 and cells were counted using Trypan Blue to assess viability and toxicity. The remaining cells were stored in RLT Lysis buffer for further DNA methylation experiments. All extracted and dried samples were dissolved in PBS (phosphate buffered saline) to make stock solutions $(50 \mathrm{mg} / \mathrm{mL})$, which were further diluted to culture concentration by culture medium.

\section{Assessment of DNA Methylation of the Foxp3 Gene Promoter}

Considering the influence of gender on methylation patterns due to X-chromosome inactivation, ${ }^{40}$ DNA from male subjects were used for studying foxp 3 methylation difference among various culture conditions. The DNA methylation status of the foxp3 gene promoter was assessed by pyrosequencing of bisulfite converted DNA. Genomic DNA was isolated from PBMCs cultured with or without SF-F2 in the presence or absence of Dex and bisulfite converted using Epitect Plus DNA Bisulfite Kit (Qiagen, MD), followed by PCR amplification of the foxp3 gene promoter. Five $\mathrm{CpG}$ residues $(-138,-126,-113$, $-77,-65)$ relative to the transcriptional start site of the foxp3 gene were chosen, and schematic representation of the foxp 3 gene and the $5 \mathrm{CpG}$ sites in the promoter region was shown in Supplementary Figure $1 .{ }^{40}$ PCR products were subjected to pyrosequencing using the PyroMark Q24 Pyrosequencing system (Qiagen, MD) using sequencing primers as shown in previous studies and listed in Supplementary Table $1 .{ }^{41,42}$ Methylation levels of PCR products at each $\mathrm{CpG}$ site were determined using PyroMark 24 run software (Qiagen, MD).

\section{Statistical Analysis}

Data were analyzed using GraphPad Prism statistical software (San Diego, CA). We performed a one-way ANOVA followed by a Bonferroni $t$-test for all pair-wise comparisons if the data were approximately normally distributed. Differences between groups were analyzed using the Wilcoxon Signed Rank Test, where data were not normally distributed. All P-values of less than 0.05 , based on a twotailed test, were considered statistically significant.

\section{Results}

\section{PBMCs from Asthmatic Subjects Exhibited Reduced IL- 10 to IL-5 Ratio in Response to Anti-CD3/28 Stimulation}

PBMCs from asthmatic subjects and healthy controls were stimulated with anti-CD3/28 Dynabeads and cytokine levels in culture supernatants measured by ELISA. In both groups, cytokine levels of IL-5 and IL-10 were significantly increased in response to anti-CD3/28 Dynabeads (Figure 2A and B, $\mathrm{p}<0.001-0.05)$. Compared with control subjects, IL-5 production was significantly higher in the asthmatic subjects $(\mathrm{p}<0.05)$ while IL-10 levels did not differ significantly between the two groups. However, the ratio of IL-10/IL-5, in response to antiCD3/28 Dynabeads, was significantly lower in the asthmatic subjects compared to controls (Figure 2C, $\mathrm{p}<0.05$ ). These results indicated that the Th2 response significantly increased, and the ratio of IL-10/IL-5 was reduced in asthmatic patients. We therefore further investigated the effects of ASHMI on the
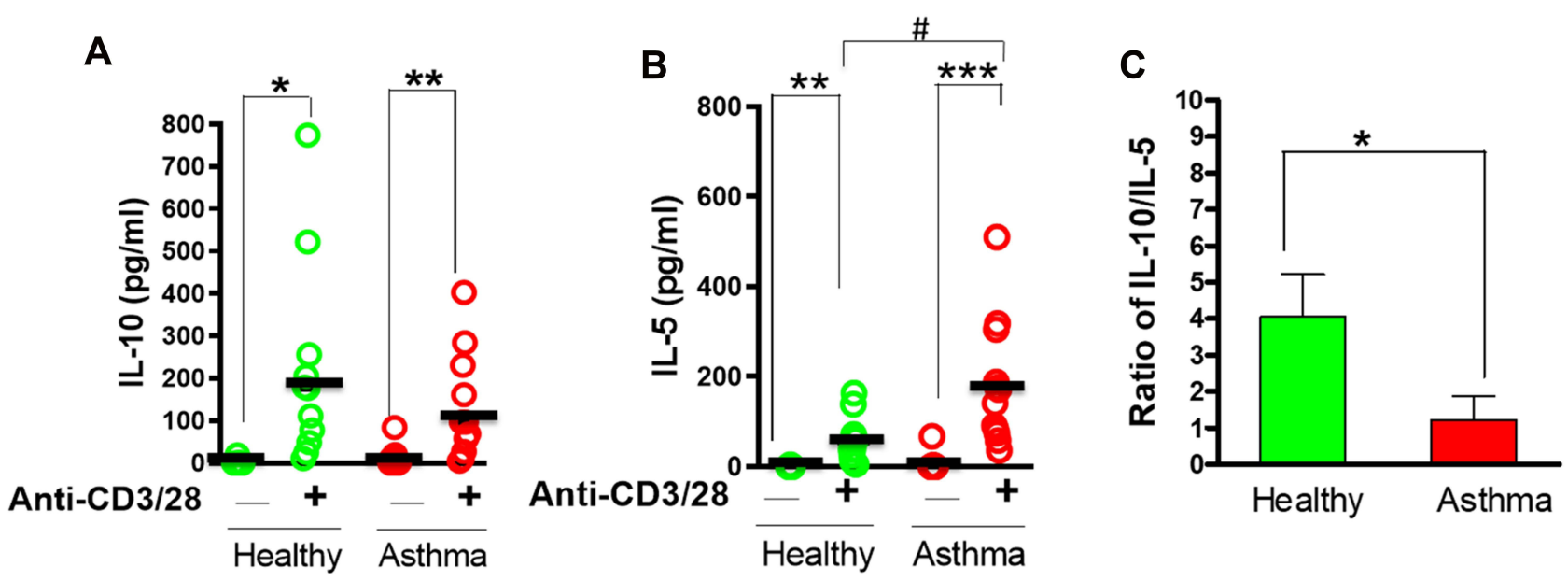

Figure 2 PBMC cytokine production and IL-10/IL-5 ratio between healthy and asthma subjects. PBMCs were stimulated with anti-CD3/28 dynabeads. * $<<0.0 .05$, ** $<<0.0$ I, $* * * p<0.001$ vs baseline; $\# p<0.05(n=11-12)$. 
production of IL-10 and IL-5 and evaluated the effect on the ratio of IL-10/IL-5 thereafter.

\section{ASHMI Caused Upregulation of IL- I0 and a Reduction of IL-5 Production in a Dose-Dependent Manner}

After 3-days of culture with ASHMI, activated PBMCs from asthmatic subjects produced less IL-5 but higher IL-10. As shown in Figure 3, when the concentration of ASHMI was increased, IL-10 production was increased in a dosedependent manner (all $\mathrm{p}<0.05$, Figure $3 \mathrm{~A}$ ). At highest concentration of ASHMI $(500 \mu \mathrm{g} / \mathrm{mL})$, the IL-10 decreased comparing with ASHMI at $125 \mu \mathrm{g} / \mathrm{mL}$. Unlike the linear dose-dependent manner, the effect of ASHMI was a bellshaped dose response. This type of dose response has been reported by other drugs. ${ }^{43,44}$ ASHMI also caused decreased IL-5 levels in a dose-dependent manner, although the $500 \mu \mathrm{g} /$ $\mathrm{mL}$ dose almost reached the statistic difference $(\mathrm{p}=0.056)$ and the $125 \mu \mathrm{g} / \mathrm{mL}$ dose was statistically significant $(\mathrm{p}<0.05$, Figure 3B). ASHMI also increased the ratio of IL-10/IL-5 production and achieved statistical significance at the $125 \mu \mathrm{g} /$

A

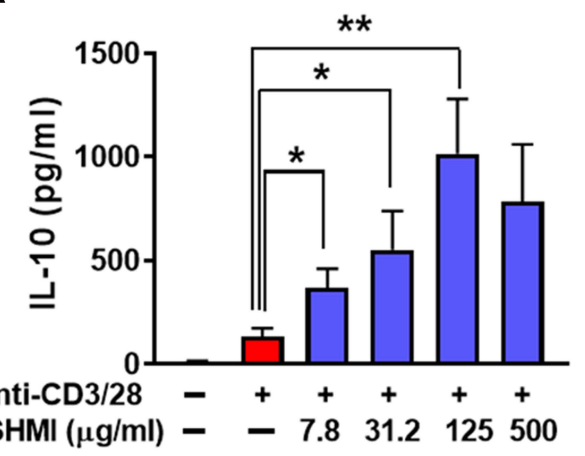

C

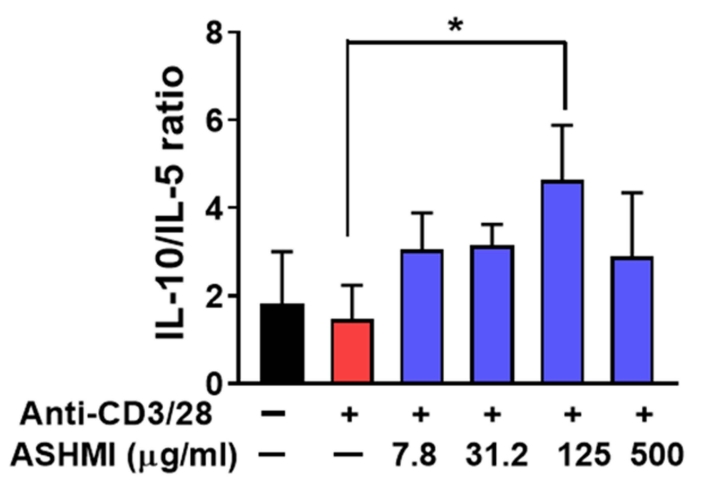

$\mathrm{mL}$ dose $(\mathrm{p}<0.05$, Figure $3 \mathrm{C})$. No cell toxicity was observed at any concentration (Figure 3D). The results proved that the ASHMI enhanced the anti-inflammatory cytokine IL-10 and restrained the production of IL- 5 to balance the Th2 activity, leading to increased IL-10/IL5 ratio.

\section{GU and SF Showed Immunomodulatory Activity on IL-10 and IL-5 Production}

ASHMI is composed of three different herbs: GU, SF, and GL. To select the main components from ASHMI, individual herb extracts were further used to evaluate their efficacy. To this end, PBMCs of asthmatic subjects were cultured with anti-CD3/28 in the presence of a specific concentration of each individual herb in ASHMI (GU, SF, and GL) that corresponds to $125 \mu \mathrm{g} / \mathrm{mL}$ dose of ASHMI. Equivalent doses were as follows: $\mathrm{GU}$ at $25.0 \mu \mathrm{g} / \mathrm{mL}, \mathrm{SF}$ at $56.3 \mu \mathrm{g} /$ $\mathrm{mL}$, and $\mathrm{GL}$ at $43.7 \mu \mathrm{g} / \mathrm{mL}$. For activated PBMC treated with GL, IL-5 and IL-10 levels in the cultures did not significantly differ from anti-CD3/28 alone, however activated PBMCs treated with GU or SF significantly increased IL-10 but decreased IL-5 productions (Figure $4 \mathrm{~A}$ and $\mathrm{B}$, all $\mathrm{p}<0.05$ ).
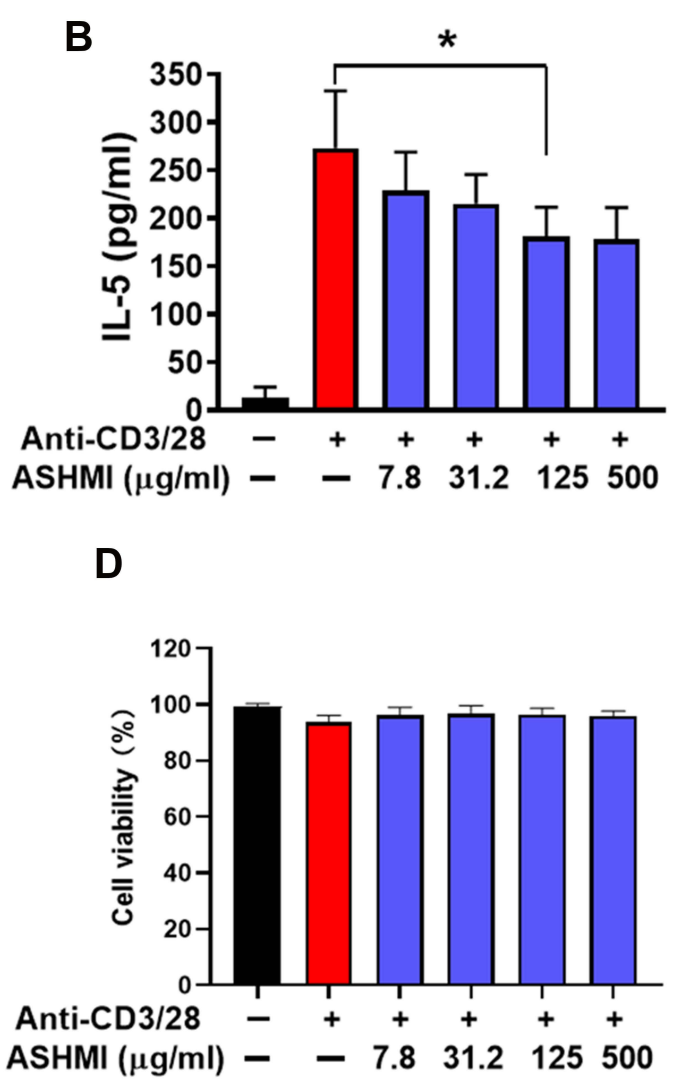

Figure 3 Effect of ASHMI on PBMC cytokine production. IL-10 (A) and IL-5 (B) cytokine production. (C) The ratio of IL-I0/IL-5. (D) Percentage of cell viability. *P<0. 0.05, $* * p<0.1(n=6)$ 
A

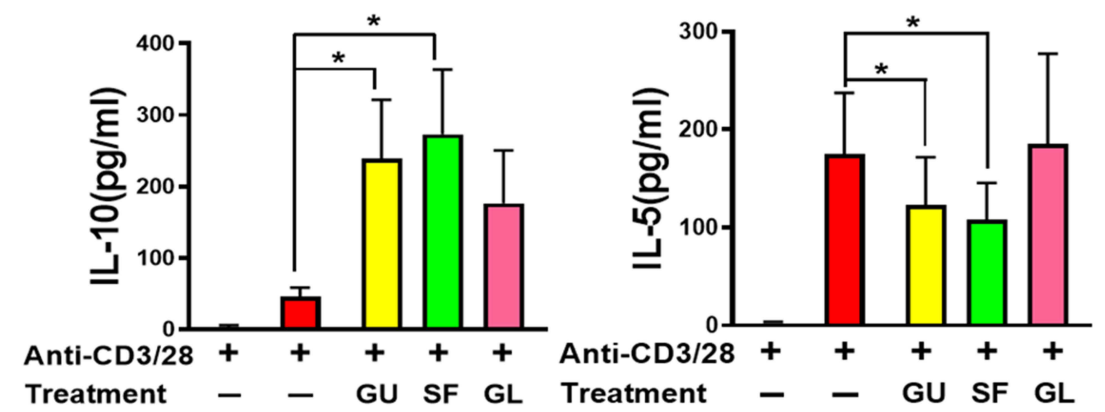

C

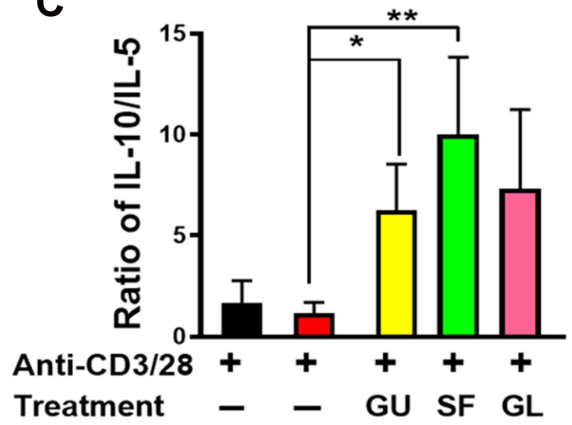

Figure 4 Effect of individual extract of herbal constituents on cytokine production of asthmatic subject PBMCs $(n=8)$. Cytokine production of IL-I0 (A) and IL-5 (B). (C) The ratio of IL-I0/IL-5 production. ${ }^{*} \mathrm{p}<0.05$, ${ }^{* *} \mathrm{p}<0.1 \quad(\mathrm{n}=8-10)$.

Furthermore, SF and GU have higher ratios of IL-10/IL-5 than other conditions and SF produced the highest ratio of IL10/IL-5 among all conditions (Figure 4C, $\mathrm{p}<0.05$ for GU, $\mathrm{p}<0.01$ for $\mathrm{SF}$ ). Therefore, SF was considered as the main herb to regulate the Th2 activity from ASHMI. The SF extract was further divided into SF-F1-4 to investigate their efficacy.

\section{SF-F2 and SF-F4 Contained the Immunomodulatory Active Compounds}

Alkaloids, flavonoids, and terpenoids have been reported as the main active compounds in $\mathrm{SF}^{45,46}$ Alkaloids show high polarity, while flavonoids and terpenoids are less polar compounds. The different polarity determined that the retention time of alkaloids is shorter than that of flavonoids and terpenoids. Fractionation and HPLC analysis revealed that SF fraction 1 (SF-F1) and SF fraction 2 (SF-F2) contained high polar compounds such as alkaloids, while SF fraction 3 (SFF3) and SF fraction 4 (SF-F4) contained less polar compounds such as flavonoids or terpenoids (Figure 1). PBMCs were cultured with each fraction. Activated PBMCs cultured with SF-F2 showed significantly increased IL-10 production compared to PBMCs cultured in anti-CD3/28 alone $(\mathrm{p}<0.05$, Figure 5A). The other three $\mathrm{SF}$ fractions did not significantly affect IL-10 levels. However, SF-F4 reduced IL-5 production in activated PBMCs ( $<0.05$, Figure 5B). Other SF fractions did not significantly affect IL-5 levels. Overall, SF-F2 increased IL-10 production by PBMCs but did not affect IL-5 production; SF-F4 decreased IL-5 production but did not affect IL-10 production. Furthermore, SF-F2 increased the ratio of IL-10/IL-5 production but not the other three fractions (Figure 5C). The results indicated that SF-F2 and SF-F4 might contain the immunomodulatory active compounds to regulate IL-10 and Th2 responses.

\section{SF-F2 Immunomodulation Selectively Counteracted Dex-Induced Suppression of IL-I 0 and Synergistically Increased the IL- I0/ IL-5 Ratio When Co-Culture with Dex}

That SF-F2 increased IL-10 production prompted us to conduct a comparison study of its effect with Dex on IL-10 production. PBMCs were cultured in the presence or absence of SF-F2 and Dex. In the presence of anti-CD3
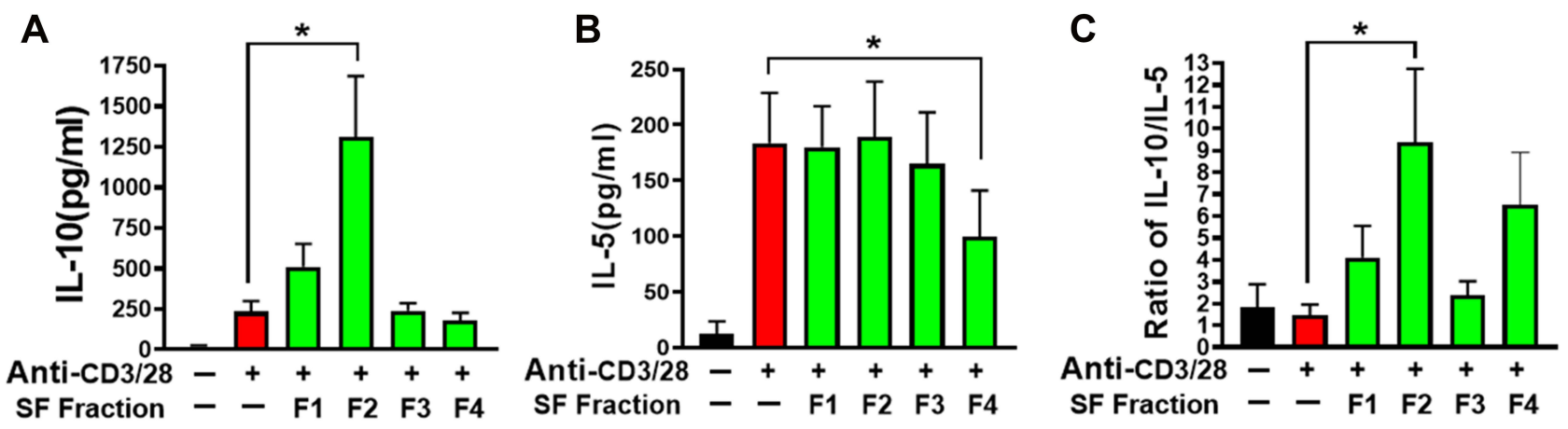

Figure 5 SF-F2 increased IL-10 and SF-F4 suppressed IL-5. (A) SF-F2 increased IL-10 production; (B) SF-F4 suppressed IL-5 production; (C) SF-F2 increased the ratio of ILI0/IL-5. ${ }^{*} \mathrm{p}<0.05(\mathrm{n}=6-8)$. 
A

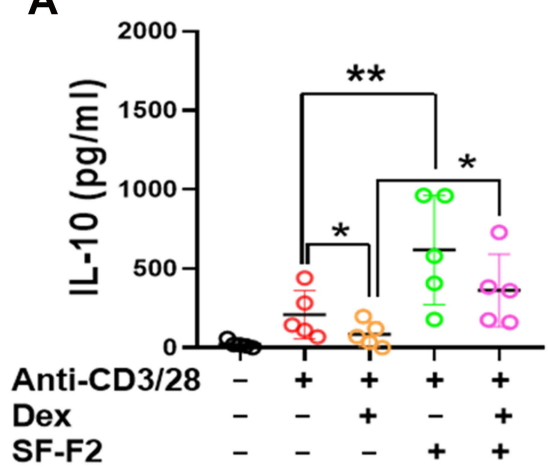

B

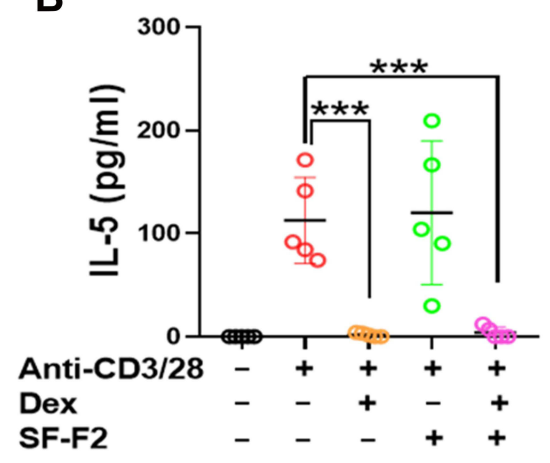

C

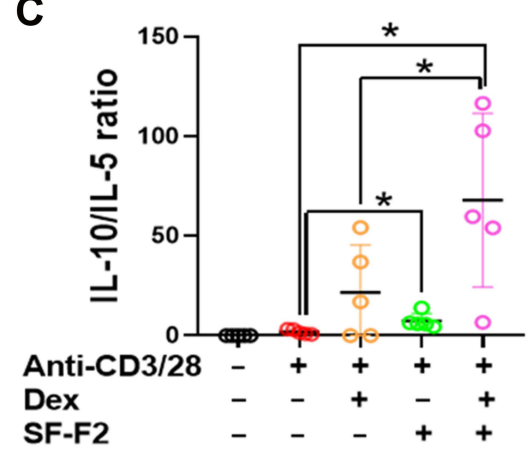

Figure 6 SF-F2 immunomodulation counteracts Dex induced-immune suppression on IL-10. The level of IL-5 (A) and IL-10 (B) were measured. The ratio of IL-10/L-5 were calculated $(\mathbf{C}) * P<0.05, * * P<0.01$, ***P<0.00I $(n=5)$.

128 , cultures with Dex had significantly lower IL-10 and IL5 than those without the Dex $(\mathrm{p}<0.001-0.05$, Figure $6 \mathrm{~A}$ and B); cultures with SF-F2 had notably higher IL-10 but had parallel IL-5 levels compared with untreated PBMCs, similar to the previous data shown in Figure 5. Importantly, in the presence of anti-CD3/28 and Dex, SF-F2 did not affect the Dex-induced IL-5 suppression compared to activated PBMCs cultured with Dex alone ( $p>0.05$, Figure $6 \mathrm{~B})$. However, SF-F2 and Dex combination selectively increased IL-10 production as compared to the untreated and Dextreated PBMCs $(\mathrm{p}<0.05$, Figure 6A). In other words, SF-F2 immunomodulation counteracted Dex-induced suppression of IL-10 but did not interfere with Dex-induced suppression of IL-5. SF-F2 generated a higher ratio of IL-10/IL-5 than untreated cells. Moreover, SF-F2 plus Dex generated the highest ratio of IL-10/IL-5 as compared to Dex-treated or SF-F2 treated PBMCs $(\mathrm{p}<0.05$, Figure $6 \mathrm{C})$, which implied that the combination of SF-F2 and Dex might exert better efficacy on the regulation of cytokines for asthmatic patients. Epigenetic regulations such as DNA methylation play a pivotal role in gene expression. It has been reported that the methylation of foxp 3 gene could regulate the Treg activity and IL-10 secretion. ${ }^{35}$ Evaluating the methylation level of foxp 3 gene provides us with a deeper understanding of the mechanisms of Dex and SF-2 in the immunomodulation of asthmatic patients.

\section{SF-F2 When Combined with Dexamethasone Reduces Foxp3 Promoter Methylation at $\mathrm{CPG}^{-126}$}

In activated PBMC cultures, it seems that no significant difference was observed about the average methylation of foxp3 $\mathrm{CpG}$ sites between different groups (Figure 7A).
However, activated PBMCs with Dex showed a high trend compared to the activated PBMCs without Dex in average methylation and the individual $5 \mathrm{CpG}$ sites (Figure 7). The potential upregulated methylation of foxp 3 by Dex indicated the immuno-functional suppression of Dex. Moreover, activated PBMCs in the presence of both Dex and SF-F2 had significantly less methylation than activated PBMCs cultured with Dex alone at $\mathrm{CpG}^{-126}$ $(\mathrm{p}<0.05$, Figure $7 \mathrm{C})$ although there were no statistical differences at other $4 \mathrm{CpG}$ sites (Figure 7B, D, E, and F). The results indicate that SF-F2 combination with Dex might downregulate the Foxp3 promoter methylation at $\mathrm{CpG}^{-126}$ to counteract Dex-induced immuno-suppression.

\section{Discussion}

A previous study demonstrated the association of reduced IL-10/IL-5 ratio with remission in asthmatic patients, and further proposed that decreased IL-5 production is due to the enhanced production of inhibitory or regulatory cytokines, such as IL-10. ${ }^{11}$ Therefore, cytokine modulation of IL-10 and IL-5 is an important area of research for the development of novel asthma therapies. ${ }^{47-50}$ In this study, we first compared the cytokine profile of asthmatic subjects to non-atopic controls and demonstrated a significant reduction of the ratios of IL-10/IL-5 in response to antiCD3/CD28 stimulation (via TCR receptor and costimulatory signaling) in asthmatic subjects compared to healthy controls. Importantly, ASHMI significantly reduced IL-5 levels and increased IL-10 production by PBMCs from asthmatic subjects in a dose-dependent manner. ASHMI significantly modulated asthmatic subject PBMCs IL-10/IL-5 ratios away from allergic reactions and towards a non-allergic phenotype. No cytotoxicity was observed in PBMCs of asthmatic subjects in this 


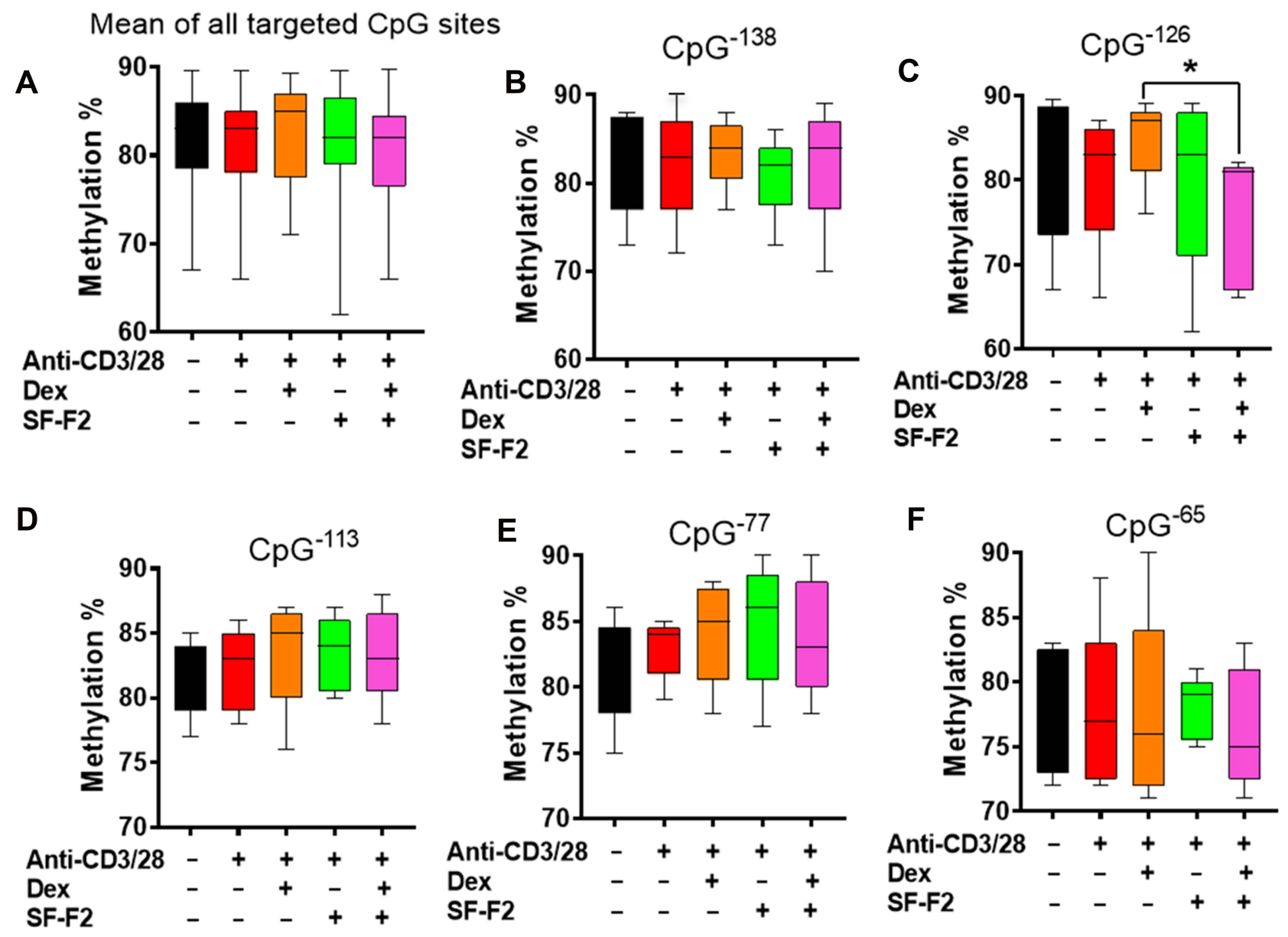

Figure 7 Percent methylation of $C_{p}$ G residues in the FOXP3 gene promoter. Percent methylation of $C_{p}$ residues in the human foxp3 promoter were assessed by pyrosequencing. ${ }^{*} \mathrm{p}<0.05(\mathrm{n}=5)$.

study. In addition, we also found that ASHMI significantly increased IFN- $\gamma$ production in response to anti-CD3/CD28 stimulation (Supplementary Figure 2), which is consistent with our previous findings that ASHMI increased IFN- $\gamma$ in human asthmatics ${ }^{27}$ and animal model of asthma. ${ }^{26}$ Given the novelty of the ASHMI enhancement of IL-10, we focused on our study to identify the components in ASHMI that are responsible for IL-10 production and the underlying mechanism.

We next attempted to delineate the activity of the individual herbal constituents responsible for IL-10 and IL-5 regulation. In an analysis of the individual herbs in their respective ASHMI dose-appropriate concentrations, we found that GU and SF, but not GL, significantly reduced IL- 5 and increased IL-10 cytokine production by PBMCs. This led to a significant alteration of the IL-10/ IL-5 ratio from 0.8 in stimulated/untreated PBMCs to 6 in stimulated/GU treated cells, and up to 9 in stimulated/SF treated cells. Statistical analysis also showed that SF- treated cells showed the most significant increase in IL10/IL-5 ratio. We therefore focused on SF and further identified that SF-F2 that contains alkaloids was most potent in increasing IL-10, whereas SF-F4 that contains triterpenoids significantly suppressed IL-5. However, only SF-F2 showed significant increases in the IL-10/IL-5 ratio. In agreement with these findings, clinical studies have demonstrated that SF alkaloid could improve respiratory function, enhance quality of life, and relieve clinical symptoms in asthma subjects. ${ }^{51}$

In light of the detrimental effects of corticosteroids (CS) on the suppression of IL-10, our findings regarding the interaction of SF-F2 with Dex are promising. SF-F2 alkaloids selectively increased IL-10 production but did not increase IL-5 production although production of both cytokines was suppressed by Dex treatment. SF-F2 exerts selective action by counteracting Dex-induced side effects of suppression of IL-10 and reserving the beneficial Dexinduced suppression of IL-5. Our current findings 
demonstrated that ASHMI, and particularly SF-F2, has an immunomodulatory capacity, even in the presence of CS, and thus could restore the usefulness of a currently common asthma treatment. Given the previous findings that corticosteroid treatments limit the development of Treg cells and further enhance subsequent $\mathrm{Th} 2$ responses and aggravate the long-term course of allergic diseases and asthma, ${ }^{20}$ our results may support the combination of SF alkaloid-rich fraction and CS as a promising therapeutic option for patients with asthma. Moreover, for the first time, we found that the SF alkaloid-rich fraction with Dex demonstrates synergistic effects of augmenting the IL-10/IL-5 ratio. Two possibilities exist regarding the augmenting of the IL-10/IL-5 ratio in the combination of SF alkaloid-rich fraction with Dex treatment. One is that SF-F2 alkaloids directly enhance or normalize production of IL-10, and the second is that the enhanced production of IL-10 serves as an inhibitory or regulatory cytokine that further suppresses IL-5 production. While many alkaloid compounds have been isolated and identified from Sophora flavescens, ${ }^{46}$ there are limited studies on immune cytokines in allergy or asthma diseases. One study from Zhou's group showed that total alkaloids of SF, which contain many different compounds like sophocarpine, matrine, oxymatrine, sophoridine, sophoramine, aloperine and cytosine, increased the expression of $\mathrm{CD} 4{ }^{+} \mathrm{CD} 25^{+}$ Tregs and IL-10 in an animal model of experimental colitis. $^{52}$ These suggested that a purified form of active compounds derived from SF-F2 combined with CS may achieve effective suppression of proinflammatory Th2 activity and restore the development of respiratory tolerance.

Traditional Chinese medicine uses complex mixtures of herbs where the active components work synergistically and removal of some components, even if inactive, can destabilize the active components. In addition, many of the components may be transformed in vivo into more complex active metabolites that could be eliminated unintentionally (Dunkin D et al, manuscript in preparation). There are at least 5 SF alkaloids identified: matrine, oxymatrine, hydroxymatrine, N-methylcytisine and anagyrine. ${ }^{51} \mathrm{We}$ tested matrine, the most common alkaloid compound from SF-F2, which has not shown the same effect as the fraction SF-F2 to increase IL-10 level or IL-10/IL-5 ratio (data not shown). We continue to purify more active compounds from SF-F2 and test their synergistic effects in various combinations, which will eventually enable the use of these compounds for the treatment of asthma.

The findings of augmented IL-10 production prompted us to address the mechanism of action. Tregs have been previously demonstrated to contribute to the maintenance of the Th1/Th2 balance, and it is known to produce IL-10 and express the transcription factor foxp3. ${ }^{35}$ Further, a recent study employed foxp3 promoter methylation as a possible indicator of Treg function and found that reduced foxp3 promoter methylation was associated with higher lung function. It was shown that exercise may protect against the harms of air pollution exposure on health in children, in part by modulating foxp3 gene promoter methylation. ${ }^{40}$ In this study, since SF-F2 plus CS induced high IL-10/IL-5 ratio, we expected that the foxp3 promoter region would be increasingly methylated in antiCD3/CD28 stimulated conditions, and less methylated in treated conditions (ie combination of Dex and SF-F2). In agreement with this expectation, the foxp 3 promoter region was relatively demethylated in PBMC cultures with both Dex and SF-F2. Interestingly, when PBMCs were cultured with Dex, methylation averaged over the five chosen sites showed a trend of higher level than the control condition (CD3/28 alone). This would suggest reduced activity of Tregs. Therefore, this finding of hypomethylation of foxp 3 promoter in addition to the high IL-10/IL-5 ratio results suggests that ASHMI, especially SF-F2, may alleviate asthma by increasing IL-10 production further regulating or inhibiting Th2-associated activation of eosinophils, while CS broadly suppresses the entire immune system. Although the results of Foxp3 promoter methylation displayed high variations, it is still convincing considering that the samples were collected from individual patients owning different genetic materials, different growing environments, and different physical qualifications.

Our results also demonstrated that ASHMI significantly increases DNA methylation of the IL-5 promoter in PBMCs from asthmatic subjects (data not shown), consistent with a previous study. ${ }^{53}$ This suggests a mechanism for the reduction in IL-5 levels seen in PBMC cultures with ASHMI and with SF-F4. IL-5 is generally accepted to be associated with Th2 activity and eosinophilic inflammation, which in turn is responsible for the clinical presentation of asthma including wheezing, breathlessness, and long-term airway remodeling. ${ }^{54}$ Anti-IL-5 treatment reduced circulating eosinophil numbers in atopic asthma patients, suggesting a role for IL-5 in the recruitment of these inflammatory cells. ${ }^{55,56}$ These prior findings, in conjunction with our current results, suggest that ASHMI, especially SF-F4 may reduce inflammation by reducing IL-5 mediated, Th2-associated activation of eosinophils. 
Our current findings demonstrated that ASHMI, and particularly SF-F2, has an immunomodulatory capacity, in terms of restoring, even in the presence of dexamethasone, a currently common asthma treatment. These results present numerous avenues - both within clinical and basic science realms, for the continuation of this study. We are approaching the identification of compounds in ASHMI that influence cytokine production, but much remains to be determined. The current study falls short of confirming which cell types in particular are responsible for the production of each cytokine in vitro. Although foxp 3 has been considered as a major transcription factor for Treg cells, other T-cells might also express foxp3. Moreover, IL-10 is a major cytokine of Treg, which might also be secreted by other cells. We cannot exclude other non-Treg cells may also contribute to IL-10 and foxp3 DNA methylation. Accordingly, one of the first next steps will be to confirm by flow cytometry, which cell types are responsible for producing IL-5 and IL-10. Further, while we have begun to investigate ASHMI-mediated epigenetic modifications related to Tregs, this topic is far broader than the scope of the current study. It is of vital interest to continue to purify and isolate ASHMI fractions to acquire single active compounds, in order to elucidate the mechanisms by which ASHMI fractions and individual active compounds regulate the cytokine balance, and to evaluate epigenetic modifications to other regions of foxp3, further promoting it to clinical study. We have included all asthmatic patients during the research time; however, most of them were mild asthmatic patients, which might bring bias into the results. In the future, we will plan the experiments by including more patients with moderate-to-severe asthma.

In conclusion, there is currently no pharmacologically active compound that can counteract CS immunosuppression, and the current study demonstrates that ASHMI has the potential to fill this gap. Continuing to purify and isolate active compounds from ASHMI will eventually enable the use of these compounds for the treatment of atopic conditions, without the numerous side effects associated with current allergy pharmacotherapies or with the dangers and barriers inherent to specific allergen immunotherapy. Furthermore, an understanding of ASHMI-mediated immunomodulation may enable the development of synthetic drugs that achieve an immune balance in allergic patients instead of the current CS-mediated overall immune suppression. ASHMI represents the possibility for novel approaches to modulate cytokine profiles that are beneficial for the treatment of chronic allergic inflammatory diseases.

\section{Acknowledgment}

We are grateful to Henry Ehrlich for reading this manuscript. The authors C. L., K. S., and X. -M. thank the partial institutional support from the Icahn School of Medicine at Mount Sinai.

\section{Author Contributions}

All authors contributed to data analysis, drafting, or revising the article, gave final approval for the version to be published, agreed to the submitted journal, and agreed to be accountable for all aspects of the work.

\section{Funding}

This manuscript was completed by a partial support from the Sean Parker Foundation and NIH/NCCIH \#1P01 AT002644725-01 to X-M Li, Henan University of Chinese Medicine to Y Song, ZZ Wang and M Miao, and key scientific research projects of colleges and universities in Henan Province (22A360003)

\section{Disclosure}

Xiu-Min Li received research support from the National Institutes of Health (NIH)/National Center for Complementary and Alternative Medicine (NCCAM); Food Allergy Research and Education (FARE) and Winston Wolkoff Integrative Medicine Fund for Allergies and Wellness, the Parker Foundation and Henan University of Chinese Medicine; received consultancy fees from FARE and Johnson \& Johnson Pharmaceutical Research \& Development, L.L.C. Bayer Global Health LLC; received royalties from UpToDate; is an Honorary Professor of Chinese Medical University, Taichung, Taiwan, and Henan University of Chinese Medicine; received travel expenses from the NCCAM, FARE and Henan University of Chinese Medicine and China Allergy Asthma College (Conference); share US patent PCT/US05/008417, PCT 14/875, 772, PCT/US2014/012306, PCT/US14/68396; PCT/US2017/ 056822) (pending); received practice compensation from the Integrative Health and Acupuncture P, US Times Technology Inc is managed by the related party; is a member of Herbs Springs, LLC, General Nutraceutical Technology, and Health Freedom LLC; Nan Yang shares US patent PCT/US14/68396, PCT 14/875, 772, PCT/ US2014/012306, PCT/US14/68396, and is a member of Health Freedom LLC. Kamal Srivastava share PCT/ US2017/056822) (pending). The other authors have declared that they have no conflict of interest. 


\section{References}

1. Lloyd CM, Hessel EM. Functions of T cells in asthma: more than just $\mathrm{T}(\mathrm{H}) 2$ cells. Nat Rev Immunol. 2010;10:838-848. doi:10.1038/ nri2870

2. Ngoc PL, Gold DR, Tzianabos AO, Weiss ST, Celedón JC. Cytokines, allergy, and asthma. Curr Opin Allergy Clin Immunol. 2005;5:161-166. doi:10.1097/01.all.0000162309.97480.45

3. Robinson DS. The role of the $\mathrm{T}$ cell in asthma. $J$ Allergy Clin Immunol. 2010;126:1081-1091;1092-1083. doi:10.1016/j. jaci.2010.06.025

4. Lloyd CM, Hawrylowicz CM. Regulatory $\mathrm{T}$ cells in asthma. Immunity. 2009;31:438-449. doi:10.1016/j.immuni.2009.08.007

5. Nakamura Y, Christodoulopoulos P, Cameron L, et al. Upregulation of the transcription factor GATA-3 in upper airway mucosa after in vivo and in vitro allergen challenge. $J$ Allergy Clin Immunol. 2000;105:1146-1152. doi:10.1067/mai.2000.107045

6. Guerra S, Lohman IC, Halonen M, Martinez FD, Wright AL. Reduced interferon gamma production and soluble CD14 levels in early life predict recurrent wheezing by 1 year of age. $\mathrm{Am}$ $J$ Respir Crit Care Med. 2004;169:70-76. doi:10.1164/rccm.20 0304-499OC

7. Barnes PJ. Targeting cytokines to treat asthma and chronic obstructive pulmonary disease. Nat Rev Immunol. 2018;18:454-466. doi:10. 1038/s41577-018-0006-6

8. Yalcin AD, Bisgin A, Gorczynski RM. IL-8, IL-10, TGF- $\beta$, and GCSF levels were increased in severe persistent allergic asthma patients with the anti-IgE treatment. Mediators Inflamm. 2012;2012:720976. doi:10.1155/2012/720976

9. Oh JW, Seroogy CM, Meyer EH, et al. CD4 T-helper cells engineered to produce IL-10 prevent allergen-induced airway hyperreactivity and inflammation. J Allergy Clin Immunol. 2002;110:460-468. doi:10.10 67/mai.2002.127512

10. Noma T, Hayashi M, Kawano Y, et al. Functional interleukin-5 activity in peripheral blood mononuclear cells from adolescents with mite antigen asthma in remission. Clin Exp Allergy. 1999;29:780-785. doi:10.1046/j.1365-2222.1999.00584.x

11. Tomiita M, Campos-Alberto E, Shima M, et al. Interleukin-10 and interleukin- 5 balance in patients with active asthma, those in remission, and healthy controls. Asia Pac Allergy. 2015;5:210-215. doi:10.5415/apallergy.2015.5.4.210

12. Chung F. Anti-inflammatory cytokines in asthma and allergy: interleukin-10, interleukin-12, interferon-gamma. Mediators Inflamm. 2001;10:51-59. doi:10.1080/09629350120054518

13. Akdis CA, Akdis M. Mechanisms of allergen-specific immunotherapy and immune tolerance to allergens. World Allergy Organ J. 2015;8:17. doi:10.1186/s40413-015-0063-2

14. Whalen KA, Legault $\mathrm{H}$, Hang $\mathrm{C}$, et al. In vitro allergen challenge of peripheral blood induces differential gene expression in mononuclear cells of asthmatic patients: inhibition of cytosolic phospholipase A2alpha overcomes the asthma-associated response. Clin Exp Allergy. 2008;38:1590-1605. doi:10.1111/j.1365-2222.2008. 03059.x

15. Akdis CA, Blaser K. Mechanisms of allergen-specific immunotherapy. Allergy. 2000;55:522-530. doi:10.1034/j.1398-9995. 2000.00120.x

16. Holgate ST, Polosa R. Treatment strategies for allergy and asthma. Nat Rev Immunol. 2008;8:218-230. doi:10.1038/nri2262

17. Cochrane MG, Bala MV, Downs KE, Mauskopf J, Ben-Joseph RH. Inhaled corticosteroids for asthma therapy: patient compliance, devices, and inhalation technique. Chest. 2000;117:542-550. doi:10.1378/chest.117.2.542

18. Klein $\mathrm{NC}$, Go $\mathrm{CH}$, Cunha BA. Infections associated with steroid use. Infect Dis Clin North Am. 2001;15:423-432,viii. doi:10.1016/S08915520(05)70154-9
19. Barnes PJ. Corticosteroids: the drugs to beat. Eur J Pharmacol. 2006;533:2-14. doi:10.1016/j.ejphar.2005.12.052

20. Stock P, Akbari O, DeKruyff RH, Umetsu DT. Respiratory tolerance is inhibited by the administration of corticosteroids. J Immunol. 2005;175:7380-7387. doi:10.4049/jimmunol.175.11.7380

21. Nawijn MC, Motta AC, Gras R, Shirinbak S, Maazi H, van Oosterhout AJ. TLR-2 activation induces regulatory $\mathrm{T}$ cells and long-term suppression of asthma manifestations in mice. PLoS One. 2013;8:e55307. doi:10.1371/journal.pone.0055307

22. Larché M, Akdis CA, Valenta R. Immunological mechanisms of allergen-specific immunotherapy. Nat Rev Immunol. 2006;6: 761-771. doi:10.1038/nri1934

23. Jutel M, Akdis M, Budak F, et al. IL-10 and TGF-beta cooperate in the regulatory $\mathrm{T}$ cell response to mucosal allergens in normal immunity and specific immunotherapy. Eur J Immunol. 2003;33:12 05-1214. doi:10.1002/eji.200322919

24. Nguyen TH, Casale TB. Immune modulation for treatment of allergic disease. Immunol Rev. 2011;242:258-271. doi:10.1111/j.1600-065X. 2011.01034.x

25. Kelly-Pieper K, Patil SP, Busse P, et al. Safety and tolerability of an antiasthma herbal Formula (ASHMI) in adult subjects with asthma: a randomized, double-blinded, placebo-controlled, dose-escalation Phase I study. J Altern Complement Med. 2009;15:735-743. doi:10.1089/acm.2008.0543

26. Srivastava K, Zhang T, Yang N, Sampson H, Li XM. Anti-Asthma Simplified Herbal Medicine Intervention-induced long-lasting tolerance to allergen exposure in an asthma model is interferon-gamma, but not transforming growth factor-beta dependent. Clin Exp Allergy. 2010;40:1678-1688. doi:10.1111/j.1365-2222.2010.03545.x

27. Wen MC, Wei $\mathrm{CH}, \mathrm{Hu} \mathrm{ZQ}$, et al. Efficacy and tolerability of anti-asthma herbal medicine intervention in adult patients with moderate-severe allergic asthma. $J$ Allergy Clin Immunol. 2005;116:517-524. doi:10.1016/j.jaci.2005.05.029

28. Hsu CH, Lu CM, Chang TT. Efficacy and safety of modified Mai-MenDong-Tang for treatment of allergic asthma. Pediatr Allergy Immunol. 2005;16:76-81. doi:10.1111/j.1399-3038.2005.00230.x

29. Chan CK, Kuo ML, Shen JJ, See LC, Chang HH, Huang JL. Ding Chuan Tang, a Chinese herb decoction, could improve airway hyper-responsiveness in stabilized asthmatic children: a randomized, double-blind clinical trial. Pediatr Allergy Immunol. 2006;17: 316-322. doi:10.1111/j.1399-3038.2006.00406.x

30. Chang TT, Huang CC, Hsu CH. Clinical evaluation of the Chinese herbal medicine formula STA-1 in the treatment of allergic asthma. Phytother Res. 2006;20:342-347. doi:10.1002/ptr.1843

31. Liu C, Yang N, Song Y, et al. Ganoderic acid C1 isolated from the anti-asthma formula, ASHMI ${ }^{\mathrm{TM}}$ suppresses TNF- $\alpha$ production by mouse macrophages and peripheral blood mononuclear cells from asthma patients. Int Immunopharmacol. 2015;27:224-231. doi:10. 1016/j.intimp.2015.05.018

32. Yang N, Liang B, Srivastava K, et al. The Sophora flavescens flavonoid compound trifolirhizin inhibits acetylcholine induced airway smooth muscle contraction. Phytochemistry. 2013;95:259-267. doi:10.1016/j.phytochem.2013.07.023

33. Liu C, Yang N, Chen X, et al. The Flavonoid 7,4'-Dihydroxyflavone Prevents Dexamethasone Paradoxical Adverse Effect on Eotaxin Production by Human Fibroblasts. Phytother Res. 2017;31:449-458. doi: $10.1002 /$ ptr.5767

34. North ML, Ellis AK. The role of epigenetics in the developmental origins of allergic disease. Ann Allergy Asthma Immunol. 2011;106:355-361; quiz 362. doi:10.1016/j.anai.2011.02.008

35. Marques CR, Costa RS, Costa GNO, et al. Genetic and epigenetic studies of FOXP3 in asthma and allergy. Asthma Res Pract. 2015;1:10. doi:10.1186/s40733-015-0012-4

36. Runyon RS, Cachola LM, Rajeshuni N, et al. Asthma discordance in twins is linked to epigenetic modifications of T cells. PLoS One. 2012;7:e48796. doi:10.1371/journal.pone.0048796 
37. Povoleri GA, Scottà C, Nova-Lamperti EA, John S, Lombardi G, Afzali B. Thymic versus induced regulatory $\mathrm{T}$ cells - who regulates the regulators? Front Immunol. 2013;4:169. doi:10.3389/fimmu.20 13.00169

38. Maruyama T, Konkel JE, Zamarron BF, Chen W. The molecular mechanisms of Foxp3 gene regulation. Semin Immunol. 2011;23:418-423. doi:10.1016/j.smim.2011.06.005

39. Srivastava KD, Dunkin D, Liu C, et al. Effect of Antiasthma Simplified Herbal Medicine Intervention on neutrophil predominant airway inflammation in a ragweed sensitized murine asthma model. Ann Allergy Asthma Immunol. 2014;112:339-347.e331-332. doi:10. 1016/j.anai.2014.01.021

40. Lovinsky-Desir S, Jung KH, Jezioro JR, et al. Physical activity, black carbon exposure, and DNA methylation in the FOXP3 promoter. Clin Epigenetics. 2017;9:65. doi:10.1186/s13148-017-0364-0

41. Lei J, Hasegawa H, Matsumoto T, Yasukawa M. Peroxisome proliferator-activated receptor $\alpha$ and $\gamma$ agonists together with TGF- $\beta$ convert human CD4+CD25- T cells into functional Foxp3+ regulatory T cells. J Immunol. 2010;185:7186-7198. doi:10.4049/jimmunol.1001437

42. Janson PC, Winerdal ME, Marits P, Thörn M, Ohlsson R, Winqvist O. FOXP3 promoter demethylation reveals the committed Treg population in humans. PLoS One. 2008;3:e1612. doi:10.1371/ journal.pone.0001612

43. Lizasoain I, Leza JC, Lorenzo P. Buprenorphine: bell-shaped dose-response curve for its antagonist effects. Gen Pharmacol. 1991;22:297-300. doi:10.1016/0306-3623(91)90452-C

44. Gallily R, Yekhtin Z. Hanu? LrOe. Overcoming the Bell-Shaped Dose-Response of Cannabidiol by Using Cannabis Extract Enriched in Cannabidiol. Pharmacol Pharmacy. 2015;6(2):11. doi:10.4236/ pp.2015.62010

45. Li JJ, Zhang X, Shen XC, et al. Phytochemistry and biological properties of isoprenoid flavonoids from Sophora flavescens Ait. Fitoterapia. 2020;143:104556. doi:10.1016/j.fitote.2020.104556

46. He X, Fang J, Huang L, Wang J, Huang X. Sophora flavescens Ait.: traditional usage, phytochemistry and pharmacology of an important traditional Chinese medicine. J Ethnopharmacol. 2015;172:10-29. doi:10.1016/j.jep.2015.06.010

47. Schülke S. Induction of Interleukin-10 Producing Dendritic Cells As a Tool to Suppress Allergen-Specific T Helper 2 Responses. Front Immunol. 2018;9:455. doi:10.3389/fimmu.2018.00455
48. Blanquiceth Y, Rodríguez-Perea AL, Tabares Guevara JH, et al. Increase of Frequency and Modulation of Phenotype of Regulatory $\mathrm{T}$ Cells by Atorvastatin Is Associated with Decreased Lung Inflammatory Cell Infiltration in a Murine Model of Acute Allergic Asthma. Front Immunol. 2016;7:620. doi:10.3389/fimmu.2016.00620

49. Trifunović J, Miller L, Debeljak Z, Horvat V. Pathologic patterns of interleukin 10 expression-a review. Biochem Med. 2015;25:36-48. doi:10.11613/BM.2015.004

50. Raeiszadeh Jahromi S, Mahesh PA, Jayaraj BS, et al. Serum levels of IL-10, IL-17F and IL-33 in patients with asthma: a case-control study. $J$ Asthma. 2014;51:1004-1013. doi:10.3109/02770903.20 14.938353

51. Zhang YB, Luo D, Yang L, et al. Matrine-Type Alkaloids from the Roots of Sophora flavescens and Their Antiviral Activities against the Hepatitis B Virus. J Nat Prod. 2018;81:2259-2265. doi:10.1021/ acs.jnatprod.8b00576

52. Zhou Y, Wang H, Liang L, Zhao WC, Chen Y, Deng HZ. Total alkaloids of Sophora alopecuroides increases the expression of CD4+ CD25+ Tregs and IL-10 in rats with experimental colitis. Am J Chin Med. 2010;38:265-277. doi:10.1142/S0192415X10 00783X

53. Barton SJ, Ngo S, Costello P, et al. DNA methylation of Th2 lineage determination genes at birth is associated with allergic outcomes in childhood. Clin Exp Allergy. 2017;47:1599-1608. doi:10.1111/ cea. 12988

54. Bousquet J, Jeffery PK, Busse WW, Johnson M, Vignola AM. Asthma. From bronchoconstriction to airways inflammation and remodeling. Am J Respir Crit Care Med. 2000;161:1720-1745. doi:10.1164/ajrccm.161.5.9903102

55. Leckie MJ, Ten Brinke A, Khan J, et al. Effects of an interleukin-5 blocking monoclonal antibody on eosinophils, airway hyper-responsiveness, and the late asthmatic response. Lancet. 2000;356:2144-2148. doi:10.1016/S0140-6736(00)03496-6

56. Farne HA, Wilson A, Powell C, Bax L, Milan SJ. Anti-IL5 therapies for asthma. Cochrane Database Syst Rev. 2017;9:CD010834. doi:10.1002/14651858.CD010834.pub3

\section{Publish your work in this journal}

The Journal of Asthma and Allergy is an international, peer-reviewed open-access journal publishing original research, reports, editorials and commentaries on the following topics: Asthma; Pulmonary physiology; Asthma related clinical health; Clinical immunology and the immunological basis of disease; Pharmacological interventions and new therapies. The manuscript management system is completely online and includes a very quick and fair peer-review system, which is all easy to use. Visit http://www.dovepress.com/testimonials.php to read real quotes from published authors. 